\title{
Unobtrusive Sensing for Home-Based Post-Stroke Rehabilitation
}

\author{
Idongesit Ekerete \\ Ulster University \\ School of Computing \\ ekerete-i@ulster.ac.uk
}

\author{
Chris Nugent \\ Ulster University \\ School of Computing \\ cd.nugent@ulster.ac.uk
}

\author{
Jim McLaughlin \\ Ulster University \\ NIBEC \\ jad.mclaughlin@ulster.ac.uk
}

\begin{abstract}
This paper proposes the fusion of low-cost unobtrusive heterogeneous sensors (MEMS thermal and radar sensors) to monitor the rehabilitation activities of post-stroke sufferers within home-based settings. Results of the proposed approach are planned to be compared with a standard EMG sensor to ascertain the authenticity, validity and repeatability of the newly introduced sensing solution.
\end{abstract}

MEMS, unobtrusive, Radar, Thermal, Sensing, Post-stroke

\section{INTRODUCTION}

Stroke is a cerebrovascular disease which interrupts the flow of oxygenated blood to certain regions of the brain. This often results in sudden loss of neurological functions making it difficult for the sufferer to carry out certain activities of daily living (ADL) (D’Aliberti et al., 2017).

Post-stroke rehabilitation has witnessed the use of Isokinetic dynamometers, low-frequency transcutaneous electrical nerve stimulation, and EMG devices, amongst others, to assist in retraining the affected muscles group(s). This is often performed within a laboratory setting, a hospital facility or through the use of wearable sensors at home. While the lab and hospital environments may seem rigorous to post-stroke patients and pose a range of logistical complexities, wearable sensors suffer from a range of problems relating to battery life, wearability and adoption. The resultant effect of these being the discontinuation of the rehabilitation process (Igual et al., 2013).

Opportunities therefore exist for the use of alternative and unobtrusive sensing solutions that can be used within home based settings. These have the potential to avoid the aforementioned issues of wearable technologies and also offer the added advantage of being able to be used within the home environment avoiding logistical issues for post stroke sufferers.

\subsection{Advantages of the Heterogeneous Fusion of Thermal and Radar Sensor}

Portable Radar sensors have come to replace the bulky Radar sensors of the past. These portable systems can provide high levels of signal to noise ratio and motion detection sensitivity, on-board signal processing capabilities and good detection range function ( $\mathrm{Li}$ et al., 2017). The UMB Radar $108 \mathrm{~A}$ is an example of a low-cost short-range radar sensor which has a stable and low current consumption rate of $200 \mathrm{~mA}(\max )$ and detection range between $1-8 \mathrm{~m}$. These capabilities make Radar sensors suitable candidates to be considered for this application compared with its ultrasonic counterpart, which has a poor resolution, low range and temperature dependability (Kelly, 2009).

A further alternative is the use of thermal sensing. As an example, the HTPA $32 \times 31$ thermal sensor is a contactless precision device capable of measuring the surface temperature of its target with the help of thermopile elements which absorb heat radiation from the target. This can be used for both stationary and moving object detections (Corelis, 2017).

While video may provide acceptable images in good lighting conditions, poor illumination can impede on the quality of images. Thermal sensors offer advantages in such situations. This is because thermal sensors in general, detect heat energy radiated from the body compared with illumination reflection by visual sensors (Kong et al., 2005).

The notion of sensor fusion provides advantages of combining the benefits of multiple sensors whilst at the same time aiming to reduce their disadvantages. Fusing for example thermal and radar sensors will provide flexible control on the data reporting format, adjustable sample rate, higher sensitivity and improved tracking performance (Kyriakides, 2016). 


\section{UNOBTRUSIVE SENSING}

Unobtrusive sensing can generally be defined as the use of sensor(s) to acquire information about target (users) without physically encountering them. It can also be referred to as remote sensing which entails gathering information about distant objects or persons without any physical connection (Zheng et al., 2014).

Unobtrusive sensing solutions have been successfully applied in many areas of science and engineering ranging from Radar and missile detection systems to the detection of structural defects and body temperature. In healthcare management and rehabilitation, its application has included detection of human emotions: blue eyes technology (Sondhi, 2017), development and evaluation of a non-obtrusive patient monitoring system with smart patient bed (Alvan Dijk et al., 2017), online and personalized daily activity recognition, habit modelling, and anomaly detection for the solitary elderly (Meng et al., 2017), temporal logic and model checking in automated recognition of human activities for ambient-assisted living (Magherini et al., 2013), and detection of workplace sedentary behaviour using thermal sensors (Synnott et al., 2016).

Moreover, unobtrusive sensing is preferred to wearable sensors in this study because it provides an on-the-go monitoring without the user having to wear or charge a device which may sometimes be forgotten (Synnott et al., 2016).

\subsection{Unobtrusive Thermal Sensing Technology}

In unobtrusive sensing sensitivity prowess of a sensor or aggregation of sensors can by no means be over-emphasized. A study by Synnott et al. (2016) considered detection of workplace sedentary behaviour using thermal sensors. A low resolution thermal sensor mounted on the ceiling was able to simultaneously monitor multiple workspaces regardless of the illumination conditions and position (sitting or standing) of workers in the room.

\subsection{Unobtrusive Radar Sensing Technology}

Advancements in semiconductor fabrication has been very useful in Radar technology. This includes a proposed cost-effective integrated Radar system which can operate at millimetre wave frequencies. Diraco et al., (2017) discussed home-based monitoring of elderly patients using a Radar-based smart sensor to detect abnormalities in ADLs. This was based on the information provided by an ultrawideband Radar sensor on locomotive and the cardiorespiratory functions of the body irrespective of physical obstruction and lighting conditions (Diraco et al., 2017).

\section{PROPOSED HETEROGENEOUS SENSOR FUSION IN POST-STROKE REHABILITATION}

The sensing solution within the proposed work aims to include the fusion of radar and thermal sensors. The $32 \times 31$ model of HTPA thermal sensors is selected for this work based on the aforementioned advantages and also that it is easy to work with and relatively inexpensive (less than 100 USD). In addition, it has high accuracy and a small footprint. Other advantages include a high signal to noise ratio because of its low noise amplifier, compact size and high sensitivity. The thermal sensor has a wide-area coverage compared to other models and can be programmed to work with Arduino microcontrollers.

The UWB 108A Radar Sensor sells for 200USD. It has a sensing distance and bandwidth of up to $8 \mathrm{~m}$ and $1 \mathrm{GHz}$, respectively. These parameters will therefore compensate for the limitation in sensing parameters of the thermal sensor on fusion. Figure 1 shows the centralized design architecture of heterogeneous sensors fusion (Panagiotis et al., 2009) considered in this work.

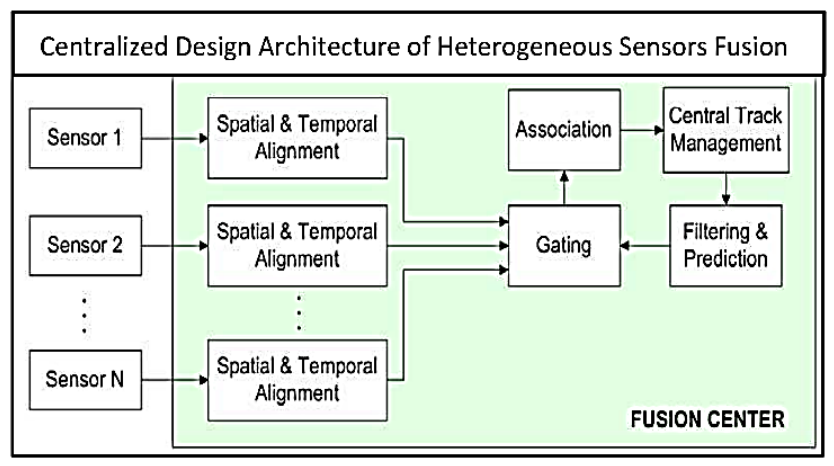

Figure 1:Centralized Design Architecture

The proposed centralized architecture (Figure 1) offers the potential for accurate data association and tracking. Other potential advantages of the design include optimization of the estimated position, object tracking precision, reduced weight, cost and power, direct implementation of algorithms and multiple hypothesis tracking.

The centralized architecture ensures correct temporal alignment of sensors before a centralized processing of raw data from each sensor.

\subsection{Measurement of Muscle Activity with the Proposed Heterogeneous Sensors Fusion}

The UWB radar sensor has a high resolution and emits short pulse signals that can penetrate fat, blood, organic tissues and bones. When these signals are incident on the target, they interact with the electromagnetic radiation emitted by the human body before being reflected back to the sensor (Wang et al., 2012).

This project will explore and decompose these reflected signals to obtain individual motor unit action potential trains (MUAPs) activated during 
neural stimulation. These signals will be amplified, rectified and filtered to obtain a refined EMG signal (Stashuk, 2001). Results will be compared with EMG sensor data obtained from wearable EMG sensor for authenticity and validity. With this information, the physiotherapist can organise an individual specific rehabilitation exercise for the patient.

Fusing the UWB radar sensor with the thermal sensor, which collects thermal radiation emitted from different parts of the human body, will help identify the actual muscle groups involved through multiscale image decomposition (MSD) algorithm. This includes averaging and continuous wavelet analysis of waveforms from regions of interest on the human body (Chekmenev et al., 2005).

\section{CONCLUSION}

In conclusion, it possible to implement a low-cost unobtrusive sensing capable of measuring the muscles activities of post-stroke patients in homebased settings using HPTA thermal and UWB Radar sensors.

\section{ACKNOWLEDGEMENTS}

This project is supported by the European Union's INTERREG VA Programme, managed by the Special EU Programmes Body (SEUPB).

\section{REFERENCES}

Alvan-Dijk et al. (2017). Development and Evaluation of a Non-obtrusive Patient Monitoring System with Smart Patient Beds. In N. Streitz \& P. Markopoulos (Eds.), Distributed, Ambient and Pervasive Interactions (pp. 482-490). Cham: Springer International Publishing.

Chekmenev et al. (2005). Non-contact, Waveletbased Measurement of Vital Signs using Thermal Imaging. Image Processing, (2005), 25-30.

Corelis. (2017). SPI Interface. Corelis 2017, 49(0), 1. Retrieved from http://www.corelis.com/education/SPI_Tutorial .htm

D'Aliberti et al. (2017). Emergency Management in Neurology, 1-91.

Diraco et al. (2017). A radar-based smart sensor for unobtrusive elderly monitoring in ambient assisted living applications. Biosensors, 7(4).

Igual et al. (2013). Challenges, issues and trends in fall detection systems. BioMedical Engineering OnLine, 12(1), 66.
Kelly, N. (2009). A Guide to Ultrasonic Sensor Set Up and Testing Instructions, Limitations, and Sample Applications, 1-7.

Kong et al. (2005). Recent advances in visual and infrared face recognition - A review. Computer Vision and Image Understanding, 97(1), 103135.

Kyriakides, I. (2016). Multiple Target Tracking Using Thermal Imaging and Radar Sensors. CoSeRa, (CoSeRa), 159-163.

Li et al. (2017). A Review on Recent Progress of Portable Short-Range Noncontact Microwave Radar Systems. IEEE Transactions on Microwave Theory and Techniques, 65(5), 1692-1706.

Magherini et al. (2013). Using Temporal Logic and Model Checking in Automated Recognition of Human Activities for Ambient-Assisted Living. Human-Machine Systems, IEEE Transactions On, 43(6), 509-521.

Meng et al. (2017). Towards online and personalized daily activity recognition, habit modeling, and anomaly detection for the solitary elderly through unobtrusive sensing. Multimedia Tools and Applications, 76(8), 10779-10799.

Panagiotis et al., L. (2009). Sensor Data Fusion in Automotive Applications, 490, 124-127.

Sondhi, A. (2017). Detecting human Emotions : Blue Eyes Technology, 2(6), 12-16.

Stashuk, D. (2001). EMG signal descomposition: how can it be accomplished an used. Journal of Electromyography and Kinesiology, 11(1), 151-173.

Synnott et al. (2016). Detection of workplace sedentary behavior using thermal sensors. Proceedings of the Annual International Conference of the IEEE Engineering in Medicine and Biology Society, EMBS, 2016Octob, 5413-5416.

Wang et al. (2012). Radar Sensing Using Ultra Wideband - Design and Implementation.

Zheng et al. (2014). Unobtrusive sensing and wearable devices for health informatics. IEEE Transactions on Biomedical Engineering, 61(5), 1538-1554. 